\title{
An inertial parallel CQ subgradient extragradient method for variational inequalities application to signal-image recovery
}

Ponkamon Kitisak ${ }^{\mathrm{a}}$, Watcharaporn Cholamjiak ${ }^{\mathrm{a}}$, Damrongsak Yambangwai ${ }^{\mathrm{a}}$

a Department of Mathematics, School of Science, University of Phayao, Phayao 56000, Thailand.

\begin{abstract}
In this paper, we introduce an inertial parallel CQ subgradient extragradient method for finding a common solutions of variational inequality problems. The novelty of this paper is using linesearch methods to find unknown $L$ constant of $L$-Lipschitz continuous mappings. Strong convergence theorem has been proved under some suitable conditions in Hilbert spaces. Finally, we show applications to signal and image recovery, and show the good efficiency of our proposed algorithm when the number of subproblems is increasing.
\end{abstract}

Keywords: CQ algorithm Subgradient extragradient method Parallel algorithm Signal recovery Image restoration.

2010 MSC: 65K15; 68W10.

\section{Introduction and Preliminaries}

Let $H$ be a real Hilbert space endowed with an inner product $\langle.,$.$\rangle and the induced norm \|$.$\| .$ A mapping A: $H \rightarrow H$ is said to be

(i) monotone if $\langle A x-A y, x-y\rangle \geq 0$ for all $x, y \in H$;

(ii) maximal monotone if it is monotone and its graph

$$
G(A):=\{(x, A x): x \in H\}
$$

is not a proper subset of one of any other monotone mapping;

Email addresses: pronkamonkitisak@gmail.com (Ponkamon Kitisak), c-wchp007@hotmail.com (Watcharaporn Cholamjiak), damrongsak. ya@up.ac.th (Damrongsak Yambangwai)

Received : May 15, 2021; Accepted: September 01, 2021; Online: September 05, 2021. 
(iii) $L$-Lipchitz continuous if there exists a positive constant $L$ such that

$$
\|A x-A y\| \leq L\|A x-A y\| \text { for all } x, y \in H .
$$

It is well-known that a monotone mapping $A: H \rightarrow H$ is maximal if and only if for each $(x, y) \in H \times H$ such that $\langle x-u, y-v\rangle \geq 0$ for all $(u, v) \in G(A)$, it follows that $y=A x$. Let $C$ be nonempty closed convex subset of $H$ and $A: H \rightarrow H$ is a nonlinear operator. The variational inequality problem (VIP) can be formulated as the problem of finding a point $x^{*} \in C$ such that

$$
\left\langle A x^{*}, x-x^{*}\right\rangle \geq 0, \forall x \in C .
$$

The set of solutions of VIP $(1)$ is denoted by $V I(A, C)$. However, the convergence of this method requires slightly strong assumptions that operators are strongly monotone or inverse strongly monotone. Many algorithms have been proposed and studied for solving VIP(1) of these algorithms involve projection methods [5, 6, 10, 11, 39, 40, 43, 46, 47, 51. The VIP(1) serves as a powerful mathematical tool and generalizes many mathematical methods, in the sense that, it includes many special problems [29] such as convex feasibility problems, linear programming problem, minimizer problem, saddle - point problems, Hierarchical variational inequality problems. It is well known that $\mathrm{VI}(\mathrm{C}, \mathrm{A})$ is equivalent to the following fixed point equation (see [2, 3, 4, 16, 17, 44, 19, 21, 23, 26, 29, 31, 32, 33]), $x=P_{C}(x-\lambda A x), \lambda>0$ and $r_{\lambda}(x):=x-P_{C}(x-\lambda A x)=0$. By using the idea of the projection method, Korelevich [24] proposed the extragradient method for solving the VIP(1) under the assumptions of Lipschitz continuous and pseudomonotone of the operator. In this method, if a closed convex set has a simple structure, then the projections onto it can be discovered easily, the extragradient method is computable and very useful. However, we have to use the projection onto $C$ into two times in the extragradient method to obtain the next approximation $x_{n+1}$ over each iteration.

Later on, Censor et al. [8] proposed the subgradient extragradient method for sloving VIP (11). The second projection onto the closed convex set of the extragradient method was replaced by the projection onto a half Space. Censor et al. [7] used the hybrid method with subgradient extragradient method for obtaining the strong convergence result. This algorithm is defined as follows:

$$
\left\{\begin{array}{l}
x_{0} \in H \\
y_{n}=P_{C}\left(x_{n}-\lambda A x_{n}\right) \\
z_{n}=\alpha_{n} x_{n}+\left(1-\alpha_{n}\right) P_{T_{n}} x_{n} \\
C_{n}=\left\{z \in H:\left\|z_{n}-z\right\| \leq\left\|x_{n}-z\right\|\right\} \\
Q_{n}=\left\{z \in H:\left\langle x_{n}-z, x_{0}-x_{n}\right\rangle \geq 0\right\} \\
x_{n+1}=P_{C_{n} \cap Q_{n}} x_{0}
\end{array}\right.
$$

Recently, Gibali [15] suggested a self-adaptive subgradient extragradient method by adopting Armijo-like searches [52] and obtained convergence result for $\mathrm{VI}(\mathrm{A}, \mathrm{C})$ in $\mathbb{R}^{n}$ when the pseudo-monotonicity and continuity of the operator are required.

Very recently, Shehu and Iyiola [34] proposed the modified viscosity algorithm with adoption of Armijoline step size rule which is called viscosity type subgradient extragradient line method for a Lipschitz continuous monotone mapping that the Lipschitz constant is unknown in an infinite dimensional Hilbert space. This method is defined as follow:

$$
\left\{\begin{array}{l}
x_{0} \in H, \\
y_{n}=P_{C}\left(x_{n}-\lambda_{n} A x_{n}\right), \lambda_{n}=\rho^{l_{n}} \\
\left(l_{n} \text { is the smallest nonnegative integer } l\right. \\
\text { such that } \left.\lambda_{n}\left\|A x_{n}-A y_{n}\right\| \leq \mu\left\|r_{\rho^{l}}\left(x_{n}\right)\right\|\right), \\
z_{n}=P_{T_{n}}\left(x_{n}-\lambda_{n} A y_{n}\right), \\
x_{n+1}=\alpha_{n} f\left(x_{n}\right)+\left(1-\alpha_{n}\right) z_{n}, n \geq 1,
\end{array}\right.
$$


where $T_{n}:=\left\{z \in H:\left\langle x_{n}-\lambda_{n} A x_{n}-y_{n}, z-y_{n}\right\rangle \leq 0\right\}, \rho, \mu \in(0,1)$ and $\left\{\alpha_{n}\right\} \subseteq(0,1)$.

Our interest in this paper is to study the common variational inequality problems (CVIP). The CVIP is to find $x^{*} \in C$ such that

$$
\left\langle A_{i} x^{*}, x-x^{*}\right\rangle \geq 0, \forall x \in C, i=1, \ldots, N,
$$

where $A_{i}: H \rightarrow H$ is a nonlinear operator for all $i=1,2, \ldots, N$.

In 2012, Censor et al. 9] presented the algorithm for solving the CVIP (4) here, finite elements are computed in parallel of each iterations. The closed convex subset $C_{n}^{1}, C_{n}^{2}, \ldots, C_{n}^{N}$ are constructed getting $x_{n+1}$ which is projected onto the intersection of these closed convex subset. This algorithm is generated by $x_{1} \in H$ and compute

$$
\left\{\begin{array}{l}
y_{n}^{i}=P_{K_{i}}\left(x_{n}-\lambda_{n}^{i} A_{i} x_{n}\right), \\
z_{n}^{i}=P_{K_{i}}\left(x_{n}-\lambda_{n}^{i} A_{i} y_{n}^{i}\right), \\
C_{n}^{i}=\left\{z \in H:\left\langle x_{n}-z_{n}^{i}, z-x_{n}-\gamma_{n}^{i}\left(z_{n}^{i}-x_{n}\right)\right\rangle \leq 0\right\} \\
C_{n}=\bigcap_{i=1}^{N} C_{n}^{i}, \\
W_{n}=\left\{z \in H:\left\langle x_{1}-x_{n}, z-x_{n}\right\rangle \leq 0\right\}, \\
x_{n+1}=P_{C_{n} \cap W_{n}} x_{1} .
\end{array}\right.
$$

This method has been extensively used due to its simplicity many authors improved it in various ways ( see $[14,18,20,25,31,35,36,37,48,49,50]$ ).

Inspired by the previous results, we introduce the new algorithm by modifying the hybrid subgradient extragradient method combining inertial technique with adoption of Armijo-line step size rule and projection onto the set of intersection sets of half-spaces to find common solution of variational inequality problems (CVIP). We prove strong convergence theorem under some suitable conditions in Hilbert spaces. Moreover, we apply our main results in image and signal recovery problems.

\section{Main Result}

In this section, we introduce an inertial parallel CQ subgradient extragradient method for variational inequalities and prove the convergence theorem of the algorithms. Let $A_{i}: H \rightarrow H$ be a family of $L_{i}$ Lipschitz continuous for all $i=1,2, \ldots, N$ with $F=\cap_{i=1}^{N} V I\left(A_{i}, C_{i}\right) \neq \phi$. The algorithm is generated as follow:

Algorithm 2.1. (Inertial parallel $C Q$ subgradient extragradient method)

Initialization: Take $\rho>0, \mu \in(0,1), \theta \in[0,1)$ and $\left\{\theta_{n}\right\} \subseteq[0, \theta]$. Select arbitrary points $x_{0}, x_{1} \in H$. For $i$ $=1,2, \ldots, N$ set $n:=1$

Step 1. Compute $s_{n}$,

$$
s_{n}=x_{n}+\theta_{n}\left(x_{n}-x_{n-1}\right) .
$$

Step 2. Compute $y_{n}$,

$$
y_{n}^{i}=P_{C}\left(s_{n}-\lambda_{n}^{i} A_{i} s_{n}\right),
$$

where $\lambda_{n}^{i}=\rho^{l^{i}}$ and $l^{i}$ is the smallest nonnegative integer such that

$$
\rho^{l^{i}}\left\|A_{i} s_{n}-A_{i} y_{n}^{i}\right\| \leq \mu\left\|s_{n}-y_{n}^{i}\right\| .
$$

Step 3. Compute $z_{n}^{i}$,

$$
z_{n}^{i}=P_{T_{n}^{i}}\left(s_{n}-\lambda_{n}^{i} A_{i} y_{n}^{i}\right), i=1, \ldots, N,
$$

where $T_{n}^{i}=\left\{v \in H:\left\langle s_{n}-\lambda_{n}^{i} A_{i} s_{n}-y_{n}^{i}, v-y_{n}^{i}\right\rangle \leq 0\right\}$.

Step 4. Compute $\bar{z}_{n}$, i.e.,

$$
\bar{z}_{n}=\operatorname{argmax}\left\{\left\|z_{n}^{i}-s_{n}\right\|: i=1, \ldots, N\right\} .
$$


Step 5. Compute $x_{n+1}=P_{C_{n} \cap Q_{n}} x_{1}$, where

$$
C_{n}=\left\{v \in H:\left\|\bar{z}_{n}-v\right\| \leq\left\|s_{n}-v\right\|\right\},
$$

and

$$
Q_{n}=\left\{v \in H:\left\langle v-x_{n}, x_{n}-x_{0}\right\rangle \geq 0\right\} .
$$

Step 6. Set $n:=n+1$ and back to Step 1 .

Lemma 2.2. For all $i=1,2, \ldots, N$, there exists a nonnegative integer $l^{i}$ satisfying (6).

Proof. Suppose $\left\|s_{n}-y_{n_{0}}^{i}\right\|=0$ for some $n_{0} \geq 1$. Take $l^{i}=n_{0}$, which satisfies (6). Suppose that $\left\|s_{n}-y_{n_{1}}^{i}\right\| \neq 0$ for some $n_{1} \geq 1$ and assume the contrary that $\rho^{n_{1}}\left\|A_{i} s_{n}-A_{i} y_{n_{1}}^{i}\right\|>\mu\left\|s_{n}-y_{n_{1}}^{i}\right\|$. Then, by Lemma 6.3 of [12] and the fact that $\rho \in(0,1)$, we obtain

$$
\begin{aligned}
\left\|A_{i} s_{n}-A_{i} y_{n_{1}}^{i}\right\| & >\frac{\mu}{\rho^{n_{1}}}\left\|s_{n}-y_{n_{1}}^{i}\right\| \\
& \geq \frac{\mu}{\rho^{n_{1}}} \min \left\{1, \rho^{n_{1}}\right\}\left\|s_{n}-y_{1}^{i}\right\| \\
& =\mu\left\|s_{n}-y_{1}^{i}\right\| .
\end{aligned}
$$

Using the fact that $P_{C}$ is continuous, we have that for all $i=1,2, \ldots, N$,

$$
y_{n_{1}}^{i}=P_{C}\left(s_{n}-\rho^{n_{1}} A_{i} s_{n}\right) \rightarrow P_{C}\left(s_{n}\right), n_{1} \rightarrow \infty .
$$

We consider two cases: $s_{n} \in C$ and $s_{n} \notin C$.

(i) If $s_{n} \in C$, then $s_{n}=P_{C}\left(s_{n}\right)$. Now, since $\left\|s_{n}-y_{n_{1}}^{i}\right\| \neq 0$ and $\rho^{n_{1}} \leq 1$, it follows from Lemma 6.3 of [12] that

$$
\begin{aligned}
0 & <\left\|s_{n}-y_{n_{1}}^{i}\right\| \\
& \leq \max \left\{1, \rho^{n_{1}}\right\}\left\|s_{n}-y_{1}^{i}\right\| \\
& =\left\|s_{n}-y_{1}^{i}\right\| .
\end{aligned}
$$

Letting $n_{1} \rightarrow \infty$ in $(7)$, we have that

$$
0=\left\|A_{i} s_{n}-A_{i} s_{n}\right\| \geq \mu\left\|s_{n}-y_{1}^{i}\right\|>0 .
$$

This is a contradiction and hence (6) is valid.

(ii) If $s_{n} \notin C$, then

$$
\rho_{n_{1}}^{i}\left\|A_{i} s_{n}-A_{i} y_{n_{1}}^{i}\right\| \rightarrow 0, n_{1} \rightarrow \infty
$$

while

$$
\begin{aligned}
\lim _{n_{1} \rightarrow \infty} \mu\left\|s_{n}-P_{C}\left(s_{n}-\rho^{n_{1}} A_{i} s_{n}\right)\right\| & =\mu \lim _{n_{1} \rightarrow \infty}\left\|s_{n}-P_{C}\left(s_{n}-\rho^{n_{1}} A_{i} s_{n}\right)\right\| \\
& =\mu\left\|s_{n}-P_{C}\left(s_{n}\right)\right\|>0 .
\end{aligned}
$$

This is a contradiction. Therefore, Algorithm 2.1 is well defined and implementable. 
Lemma 2.3. Suppose that $x^{*} \in F$ and the sequences $\left\{y_{n}^{i}\right\},\left\{z_{n}^{i}\right\}$ generated by Step 1 and Step 2 of Algorithm 2.1. Then

$$
\begin{aligned}
\left\|z_{n}^{i}-x^{*}\right\|^{2} \leq & \left\|x_{n}-x^{*}\right\|^{2}+(1+c) 2 \theta_{n}\left\langle x_{n}-x_{n-1}, y_{n}^{i}-x^{*}\right\rangle \\
& -c\left(\left\|x_{n}-y_{n}^{i}\right\|^{2}+\left\|z_{n}^{i}-y_{n}^{i}\right\|^{2}\right)
\end{aligned}
$$

where $c=1-\mu>0$.

Proof. Let $x^{*} \in F$. For each $\mathrm{i}=1,2, \ldots \mathrm{N}$, let $u_{n}^{i}=s_{n}-\lambda_{n}^{i} A_{i} y_{n}^{i}, \forall n \geq 1$, we have

$$
\begin{aligned}
\left\|z_{n}^{i}-x^{*}\right\|^{2} & =\left\|P_{T_{n}^{i}}\left(s_{n}-\lambda_{n}^{i} A_{i} y_{n}^{i}\right)-x^{*}\right\|^{2} \\
& =\left\|P_{T_{n}^{i}}\left(u_{n}^{i}\right)-x^{*}\right\|^{2} \\
& =\left\|\left(P_{T_{n}^{i}}\left(u_{n}^{i}\right)-u_{n}^{i}\right)+\left(u_{n}^{i}-x^{*}\right)\right\|^{2} \\
& =\left\|u_{n}^{i}-x^{*}\right\|^{2}+\left\|u_{n}^{i}-P_{T_{n}^{i}}\left(u_{n}^{i}\right)\right\|^{2}+2\left\langle P_{T_{n}^{i}}\left(u_{n}^{i}\right)-u_{n}^{i}, u_{n}^{i}-x^{*}\right\rangle
\end{aligned}
$$

since $x^{*} \in F \subseteq C \subseteq T_{n}^{i}$ by the property of the metric projection $P_{T_{n}^{i}}$, we derive

$$
\begin{array}{r}
2\left\|u_{n}^{i}-P_{T_{n}^{i}}\left(u_{n}^{i}\right)\right\|^{2}+2\left\langle P_{T_{n}^{i}}\left(u_{n}^{i}\right)-u_{n}^{i}, u_{n}^{i}-x^{*}\right\rangle \\
=2\left\langle u_{n}^{i}-P_{T_{n}^{i}}\left(u_{n}^{i}\right), x^{*}-P_{T_{n}^{i}}\left(u_{n}^{i}\right)\right\rangle \leq 0
\end{array}
$$

and

$$
\left\|u_{n}^{i}-P_{T_{n}^{i}}\left(u_{n}^{i}\right)\right\|^{2}+2\left\langle P_{T_{n}^{i}}\left(u_{n}^{i}\right)-u_{n}^{i}, u_{n}^{i}-x^{*}\right\rangle \leq-\left\|u_{n}^{i}-P_{T_{n}^{i}}\left(u_{n}^{i}\right)\right\|^{2} .
$$

We then obtain from Algorithm 2.1] and Lemma 2.3 (ii) of [42] that

$$
\begin{aligned}
\left\|z_{n}^{i}-x^{*}\right\|^{2} & \leq\left\|u_{n}^{i}-x^{*}\right\|^{2}-\left\|u_{n}^{i}-P_{T_{n}^{i}}\left(u_{n}^{i}\right)\right\|^{2} \\
& =\left\|\left(s_{n}-\lambda_{n}^{i} A_{i} y_{n}^{i}\right)-x^{*}\right\|^{2}-\left\|\left(s_{n}-\lambda_{n}^{i} A_{i} y_{n}^{i}\right)-z_{n}^{i}\right\|^{2} \\
& =\left\|s_{n}-x^{*}\right\|^{2}-\left\|s_{n}-z_{n}^{i}\right\|^{2}+2 \lambda_{n}^{i}\left\langle x^{*}-z_{n}^{i}, A_{i} y_{n}^{i}\right\rangle .
\end{aligned}
$$

Since $A_{i}$ is the monotone operator for all $\mathrm{i}=1,2, \ldots, \mathrm{N}$, we have

$$
\begin{aligned}
0 & \leq\left\langle A_{i} y_{n}^{i}-A_{i} x^{*}, y_{n}^{i}-x^{*}\right\rangle \\
& =\left\langle A_{i} y_{n}^{i}, y_{n}^{i}-x^{*}\right\rangle-\left\langle A_{i} x^{*}, y_{n}^{i}-x^{*}\right\rangle \\
& \leq\left\langle A_{i} y_{n}^{i}, y_{n}^{i}-x^{*}\right\rangle \\
& =\left\langle A_{i} y_{n}^{i}, y_{n}^{i}-z_{n}^{i}+z_{n}^{i}-x^{*}\right\rangle \\
& =\left\langle A_{i} y_{n}^{i}, y_{n}^{i}-z_{n}^{i}\right\rangle+\left\langle A_{i} y_{n}^{i}, z_{n}^{i}-x^{*}\right\rangle .
\end{aligned}
$$

Thus,

$$
\left\langle x^{*}-z_{n}^{i}, A_{i} y_{n}^{i},\right\rangle \leq\left\langle A_{i} y_{n}^{i}, y_{n}^{i}-z_{n}^{i}\right\rangle
$$

Using 12 in (13), we obtain

$$
\begin{aligned}
\left\|z_{n}^{i}-x^{*}\right\|^{2} \leq & \left\|s_{n}-x^{*}\right\|^{2}-\left\|s_{n}-z_{n}^{i}\right\|^{2}+2 \lambda_{n}^{i}\left\langle A_{i} y_{n}^{i}, y_{n}^{i}-z_{n}^{i}\right\rangle \\
= & \left\|s_{n}-x^{*}\right\|^{2}+2 \lambda_{n}^{i}\left\langle A_{i} y_{n}^{i}, y_{n}^{i}-z_{n}^{i}\right\rangle-\left\|s_{n}-y_{n}^{i}+y_{n}^{i}-z_{n}^{i}\right\|^{2} \\
= & \left\|s_{n}-x^{*}\right\|^{2}+2 \lambda_{n}^{i}\left\langle A_{i} y_{n}^{i}, y_{n}^{i}-z_{n}^{i}\right\rangle-2\left\langle s_{n}-y_{n}^{i}, y_{n}^{i}-z_{n}^{i}\right\rangle \\
& -\left\|s_{n}-y_{n}^{i}\right\|^{2}-\left\|y_{n}^{i}-z_{n}^{i}\right\|^{2} \\
= & \left\|s_{n}-x^{*}\right\|^{2}+2\left\langle s_{n}-\lambda_{n}^{i} A_{i} y_{n}^{i}-y_{n}^{i}, z_{n}^{i}-y_{n}^{i}\right\rangle-\left\|s_{n}-y_{n}^{i}\right\|^{2} \\
& -\left\|y_{n}^{i}-z_{n}^{i}\right\|^{2} .
\end{aligned}
$$


Observe that

$$
\begin{aligned}
\left\langle s_{n}-\lambda_{n}^{i} A_{i} y_{n}^{i}-y_{n}^{i}, z_{n}^{i}-y_{n}^{i}\right\rangle= & \left\langle s_{n}-\lambda_{n}^{i} A_{i} s_{n}-y_{n}^{i}, z_{n}-y_{n}^{i}\right\rangle \\
& +\left\langle\lambda_{n}^{i} A_{i} s_{n}-\lambda_{n}^{i} A_{i} y_{n}^{i}, z_{n}^{i}-y_{n}^{i}\right\rangle \\
\leq & \left\langle\lambda_{n}^{i} A_{i} s_{n}-\lambda_{n}^{i} A_{i} y_{n}^{i}, z_{n}^{i}-y_{n}^{i}\right\rangle .
\end{aligned}
$$

Using the last inequality in (14, we have that

$$
\begin{aligned}
\left\|z_{n}^{i}-x^{*}\right\|^{2} \leq & \left\|s_{n}-x^{*}\right\|^{2}+2\left\langle\lambda_{n}^{i} A_{i} s_{n}-\lambda_{n}^{i} A_{i} y_{n}^{i}, z_{n}^{i}-y_{n}^{i}\right\rangle-\left\|s_{n}-y_{n}^{i}\right\|^{2} \\
& -\left\|y_{n}^{i}-z_{n}^{i}\right\|^{2} \\
= & \left\|s_{n}-x^{*}\right\|^{2}+2 \lambda_{n}^{i}\left\langle A_{i} s_{n}-A_{i} y_{n}^{i}, z_{n}^{i}-y_{n}^{i}\right\rangle-\left\|s_{n}-y_{n}^{i}\right\|^{2} \\
& -\left\|y_{n}^{i}-z_{n}^{i}\right\|^{2} \\
\leq & \left\|s_{n}-x^{*}\right\|^{2}+2 \lambda_{n}^{i}\left\|A_{i} s_{n}-A_{i} y_{n}^{i}\right\|\left\|z_{n}^{i}-y_{n}^{i}\right\|-\left\|s_{n}-y_{n}^{i}\right\|^{2} \\
& -\left\|y_{n}^{i}-z_{n}^{i}\right\|^{2} \\
\leq & \left\|s_{n}-x^{*}\right\|^{2}+2 \mu\left\|s_{n}-y_{n}^{i}\right\|\left\|z_{n}^{i}-y_{n}^{i}\right\|-\left\|s_{n}-y_{n}^{i}\right\|^{2} \\
& -\left\|y_{n}^{i}-z_{n}^{i}\right\|^{2} \\
\leq & \left\|s_{n}-x^{*}\right\|^{2}+\mu\left(\left\|s_{n}-y_{n}^{i}\right\|^{2}+\left\|z_{n}^{i}-y_{n}^{i}\right\|^{2}\right)-\left\|s_{n}-y_{n}^{i}\right\|^{2} \\
& -\left\|y_{n}^{i}-z_{n}^{i}\right\|^{2} \\
\leq & \left\|s_{n}-x^{*}\right\|^{2}+\left(\mu\left\|s_{n}-y_{n}^{i}\right\|^{2}-\left\|s_{n}-y_{n}^{i}\right\|^{2}\right) \\
& +\left(\mu\left\|z_{n}^{i}-y_{n}^{i}\right\|^{2}-\left\|y_{n}^{i}-z_{n}^{i}\right\|^{2}\right) \\
= & \left\|s_{n}-x^{*}\right\|^{2}-(1-\mu)\left\|s_{n}-y_{n}^{i}\right\|^{2}-(1-\mu)\left\|y_{n}^{i}-z_{n}^{i}\right\|^{2} \\
\leq & \left\|s_{n}-x^{*}\right\|^{2}-(1-\mu)\left(\left\|s_{n}-y_{n}^{i}\right\|^{2}+\left\|y_{n}^{i}-z_{n}^{i}\right\|^{2}\right) \\
\leq & \left\|s_{n}-x^{*}\right\|^{2}-c\left(\left\|s_{n}-y_{n}^{i}\right\|^{2}+\left\|y_{n}^{i}-z_{n}^{i}\right\|^{2}\right) .
\end{aligned}
$$

From 15 and $s_{n}=x_{n}+\theta_{n}\left(x_{n}-x_{n-1}\right)$, we have

$$
\begin{aligned}
\left\|z_{n}^{i}-x^{*}\right\|^{2} \leq & \left\|\left(x_{n}+\theta_{n}\left(x_{n}-x_{n-1}\right)\right)-x^{*}\right\|^{2} \\
& -c\left(\left\|\left(x_{n}+\theta_{n}\left(x_{n}-x_{n-1}\right)\right)-y_{n}^{i}\right\|^{2}+\left\|y_{n}^{i}-z_{n}^{i}\right\|^{2}\right) \\
\leq & \left\|\left(x_{n}-x^{*}\right)+\theta_{n}\left(x_{n}-x_{n-1}\right)\right\|^{2} \\
& -c\left(\left\|\left(x_{n}-y_{n}^{i}\right)+\theta_{n}\left(x_{n}-x_{n-1}\right)\right\|^{2}+\left\|y_{n}^{i}-z_{n}^{i}\right\|^{2}\right) \\
\leq & \left\|x_{n}-x^{*}\right\|^{2}+2\left\langle\theta_{n}\left(x_{n}-x_{n-1}\right), x_{n}-x^{*}+\theta_{n}\left(x_{n}-x_{n-1}\right)\right\rangle \\
& -c\left(\left\|x_{n}-y_{n}^{i}\right\|^{2}+2\left\langle\theta_{n}\left(x_{n}-x_{n-1}\right), x_{n}-y_{n}^{i}+\theta_{n}\left(x_{n}-x_{n-1}\right)\right\rangle\right. \\
& \left.+\left\|y_{n}^{i}-z_{n}^{i}\right\|^{2}\right) \\
\leq & \left\|x_{n}-x^{*}\right\|^{2}+2 \theta_{n}\left\langle x_{n}-x_{n-1}, s_{n}-x^{*}\right\rangle \\
& -c\left(\left\|x_{n}-y_{n}^{i}\right\|^{2}+2 \theta_{n}\left\langle x_{n}-x_{n-1}, s_{n}-y_{n}^{i}\right\rangle+\left\|y_{n}^{i}-z_{n}^{i}\right\|^{2}\right) \\
\leq & \left\|x_{n}-x^{*}\right\|^{2}-c\left(\left\|x_{n}-y_{n}^{i}\right\|^{2}+\left\|y_{n}^{i}-z_{n}^{i}\right\|^{2}\right) \\
& +2 \theta_{n}\left\langle x_{n}-x_{n-1}, s_{n}-x^{*}\right\rangle+2 \theta_{n} c\left\langle x_{n}-x_{n-1}, s_{n}-y_{n}^{i}\right\rangle \\
\leq & \left\|x_{n}-x^{*}\right\|^{2}-c\left(\left\|x_{n}-y_{n}^{i}\right\|^{2}+\left\|y_{n}^{i}-z_{n}^{i}\right\|^{2}\right) \\
& +(1+c) 2 \theta_{n}\left\langle x_{n}-x_{n-1}, y_{n}^{i}-x^{*}\right\rangle .
\end{aligned}
$$


From 12 and (16), we obtain inequality (8).

Lemma 2.4. Suppose that $\left\{x_{n}\right\},\left\{y_{n}^{i}\right\},\left\{z_{n}^{i}\right\}$ generated by Algorithm 2.1. Then

(i) $F \subset C_{n} \cap Q_{n}$ and $x_{n+1}$ is well-defined for all $n \geq 0$.

(ii) If $\Sigma \theta_{n}\left\|x_{n}-x_{n-1}\right\|<\infty$, then for each $i=1, \ldots, N$, the following relations hold:

$$
\lim _{n \rightarrow \infty}\left\|x_{n+1}-x_{n}\right\|=\lim _{n \rightarrow \infty}\left\|y_{n}^{i}-x_{n}\right\|=\lim _{n \rightarrow \infty}\left\|z_{n}^{i}-x_{n}\right\|=\lim _{n \rightarrow \infty}\left\|s_{n}-y_{n}^{i}\right\|=0 .
$$

Proof. (i) Since $A_{i}$ is Lipschitz continuous, $A_{i}$ is continuous. Thus, Lemma 2.1 of [38] ensures that $V I\left(A_{i}, C\right)$ is closed and convex for all $i=1, \ldots, N$. Hence, $F$ is closed and convex. From the definitions of $C_{n}$ and $Q_{n}$, we see that $Q_{n}$ is closed and convex and $C_{n}$ is closed. On the other hand, the relation $\left\|\bar{z}_{n}-v\right\| \leq\left\|s_{n}-v\right\|$ is equivalent to

$$
2\left\langle v, s_{n}-\bar{z}_{n}\right\rangle \leq\left\|s_{n}\right\|^{2}-\left\|\bar{z}_{n}\right\|^{2} .
$$

This implies that $C_{n}$ is convex. Moreover, for each $u \in F$, from Lemma 2.3, we obtain $\left\|\bar{z}_{n}-u\right\| \leq\left\|s_{n}-u\right\|$. Thus, $F \subset C_{n}$ for all $n \geq 1$. Next, we will show that $F \subset C_{n} \cap Q_{n}$ by the induction. Indeed, $F \subset Q_{n}$ and so $F \subset C_{n} \cap Q_{n}$. Assume that $F \subset C_{n} \cap Q_{n}$ for some $n \geq 1$. From $x_{n+1}=P_{C_{n} \cap Q_{n}} x_{1}$ and the characterization of the metric projection by Lemma 2.3 (iii) of [42, we obtain

$$
\left\langle v-x_{n+1}, x_{n+1}-x_{1}\right\rangle \geq 0, \quad \forall v \in C_{n} \cap Q_{n} .
$$

Since $F \subset C_{n} \cap Q_{n},\left\langle v-x_{n+1}, x_{n+1}-x_{1}\right\rangle \geq 0$ for all $v \in F$. This together with the definition of $Q_{n+1}$ implies that $F \subset Q_{n+1}$. Thus, by the induction $F \subset C_{n} \cap Q_{n}$ for all $n \geq 1$. Since $F \neq \phi, P_{F} x_{1}$ and $x_{n+1}=P_{C_{n} \cap Q_{n}} x_{1}$ are well defined.

(ii). We have $x_{n}=P_{Q_{n}} x_{1}$ and $F \subset Q_{n}$. For each $u \in F$, by the property of the projection $P_{Q_{n}}$ we have

$$
\left\|x_{n}-x_{1}\right\| \leq\left\|u-x_{1}\right\|, \quad \forall n \geq 0 .
$$

Thus, the sequence $\left\{\left\|x_{n}-x_{1}\right\|\right\}$ is bouned and so $\left\{x_{n}\right\}$ is also bounded. From $x_{n+1} \in Q_{n}$ and $x_{n}=P_{Q_{n}} x_{1}$, we also obtain

$$
\left\|x_{n}-x_{1}\right\| \leq\left\|x_{n+1}-x_{1}\right\|, \quad \forall n \geq 0 .
$$

This implies that the sequence $\left\{\left\|x_{n}-x_{1}\right\|\right\}$ is nondecreasing. $\lim _{n \rightarrow \infty}\left\|x_{n}-x_{1}\right\|$ exists. It follows from $x_{n+1} \in Q_{n}$ and $x_{n}=P_{Q_{n}} x_{1}$, that

$$
\left\|x_{n}-x_{n+1}\right\|^{2} \leq\left\|x_{n+1}-x_{1}\right\|^{2}-\left\|x_{n}-x_{1}\right\|^{2} .
$$

From this inequality, taking $n \rightarrow \infty$, we get

$$
\lim _{n \rightarrow \infty}\left\|x_{n+1}-x_{n}\right\|=0 .
$$

By the definition of $C_{n}$ and $x_{n+1} \in C_{n}$, we have

$$
\begin{aligned}
\left\|\bar{z}_{n}-x_{n+1}\right\| & \leq\left\|x_{n+1}-s_{n}\right\| \\
& \leq\left\|x_{n+1}-x_{n}\right\|+\theta_{n}\left\|x_{n}-x_{n-1}\right\| .
\end{aligned}
$$

From the definition of $\left\{\theta_{n}\right\}$ in Step 1 and 19$)$ we have

$$
\lim _{n \rightarrow \infty}\left\|\bar{z}_{n}-x_{n+1}\right\|=0 .
$$


This together with the triangle inequality $\left\|\bar{z}_{n}-x_{n}\right\| \leq\left\|\bar{z}_{n}-x_{n+1}\right\|+\left\|x_{n+1}-x_{n}\right\|$ implies that

$$
\lim _{n \rightarrow \infty}\left\|\bar{z}_{n}-x_{n}\right\|=0
$$

From (21) and the definition of $i_{n}$, we get

$$
\lim _{n \rightarrow \infty}\left\|z_{n}^{i}-x_{n}\right\|=0, \quad \forall i=1, \ldots, N .
$$

From Lemma 2.3 and the triangle inequality, for each $u \in F$, one has

$$
c\left\|x_{n}-y_{n}^{i}\right\|^{2} \leq\left\|x_{n}-u\right\|^{2}-\left\|z_{n}^{i}-u\right\|^{2}+(2-\mu) 2 \theta_{n}\left\langle x_{n}-x_{n-1}, y_{n}^{i}-u\right\rangle
$$

From 22, 25 and the boundedness of $\left\{s_{n}\right\},\left\{x_{n}\right\},\left\{y_{n}^{i}\right\},\left\{z_{n}^{i}\right\}$ and the condition $\Sigma \theta_{n}\left\|x_{n}-x_{n-1}\right\|<\infty$, we get

$$
\lim _{n \rightarrow \infty}\left\|y_{n}^{i}-x_{n}\right\|=0, \quad i=1, \ldots, N
$$

From (15), we have

$$
\begin{aligned}
c\left\|s_{n}-y_{n}^{i}\right\|^{2} & \leq\left\|s_{n}-x^{*}\right\|^{2}-\left\|z_{n}^{i}-x^{*}\right\|^{2} \\
& =\left\|\left(x_{n}-x^{*}\right)+\theta_{n}\left(x_{n}-x_{n-1}\right)\right\|^{2}-\left\|z_{n}^{i}-x^{*}\right\|^{2} \\
& =\left\|x_{n}-x^{*}\right\|^{2}+2 \theta_{n}\left\langle x_{n}-x_{n-1}, s_{n}-x^{*}\right\rangle-\left\|z_{n}^{i}-x^{*}\right\|^{2} .
\end{aligned}
$$

From the condition $\Sigma \theta_{n}\left\|x_{n}-x_{n-1}\right\|<\infty$ and $(22)$, we get

$$
\lim _{n \rightarrow \infty}\left\|s_{n}-y_{n}^{i}\right\|=0
$$

for all $\mathrm{i}=1, \ldots, \mathrm{N}$.

Theorem 2.5. Let $C$ be a closed and convex subset of a real Hilbert space $H$. Suppose that $\left\{A_{i}\right\}_{i=1}^{N}: H \rightarrow H$ is a finite family of monotone mappings. In addition, the solution set $F$ is nonempty and $\Sigma \theta_{n}\left\|x_{n}-x_{n-1}\right\|<\infty$. Then, the sequences $\left\{x_{n}\right\},\left\{y_{n}^{i}\right\},\left\{z_{n}^{i}\right\}$ generated by Algorithm 2.1 converge strongly to $P_{F} x_{1}$.

Proof. By Lemma 2.4 $F, C_{n}, Q_{n}$ are nonempty closed and convex subsets. Besides, $F \subset C_{n} \cap Q_{n}$ for all $n \geq 1$. Therefore, $P_{F} x_{1}, P_{C_{n} \cap Q_{n}} x_{1}$ are well-defined. From Lemma 2.4. $\left\{x_{n}\right\}$ is bounded. Assume that $p$ is a weak cluster point of $\left\{x_{n}\right\}$ and $\left\{x_{n_{k}}\right\}$ is subsequence of $\left\{x_{n}\right\}$ converging weakly to $p$. Since $\left\|y_{n_{k}}^{i}-x_{n_{k}}\right\| \rightarrow 0, y_{n_{k}}^{i} \rightarrow p$. Now we prove that $p \in F$. Indeed, Lemma 2.3 of [42], ensures that the mapping

$$
Q_{i} x= \begin{cases}A_{i} x+N_{C}(x) & \text { if } x \in C, \\ \emptyset & \text { if } x \notin C,\end{cases}
$$

is maximal monotone, where $N_{C}(x)$ is the normal cone to $C$ at $x \in C$. For all $(x, y)$ in the graph of $Q_{i}$, i.e., $(x, y) \in G\left(Q_{i}\right)$, we have $y-A_{i} x \in N_{C}(x)$. By the definition of $N_{C}(x)$, we find that

$$
\left\langle x-z, y-A_{i} x\right\rangle \geq 0
$$

for all $z \in C$. Since $y_{n_{k}}^{i} \in C$,

$$
\left\langle x-y_{n_{k}}^{i}, y-A_{i} x\right\rangle \geq 0
$$

Therefore,

$$
\left\langle x-y_{n_{k}}^{i}, y\right\rangle \geq\left\langle x-y_{n_{k}}^{i}, A_{i} x\right\rangle
$$


Taking into account $y_{n_{k}}^{i}=P_{C}\left(s_{n_{k}}-\lambda_{n_{k}}^{i} A_{i} s_{n_{k}}\right)$ and Lemma 6.6 of [1], we get

$$
\begin{aligned}
\left\langle x-y_{n_{k}}^{i}, y_{n_{k}}^{i}-s_{n_{k}}+\lambda_{n_{k}}^{i} A_{i} s_{n_{k}}\right\rangle & \geq 0 \\
\left\langle x-y_{n_{k}}^{i}, \frac{y_{n_{k}}^{i}-s_{n}}{\lambda_{n_{k}}^{i}}+A_{i} s_{n_{k}}\right\rangle & \geq 0
\end{aligned}
$$

or

$$
\left\langle x-y_{n_{k}}^{i}, A_{i} s_{n_{k}}\right\rangle \geq\left\langle x-y_{n_{k}}^{i}, \frac{s_{n_{k}}-y_{n_{k}}^{i}}{\lambda_{n_{k}}^{i}}\right\rangle
$$

Therefore, from (27), 28) and the monotonicity of $A_{i}$, we find that

$$
\begin{aligned}
\left\langle x-y_{n_{k}}^{i}, y\right\rangle \geq & \left\langle x-y_{n_{k}}^{i}, A_{i} x\right\rangle \\
= & \left\langle x-y_{n_{k}}^{i}, A_{i} x-A_{i} y_{n_{k}}^{i}\right\rangle+\left\langle x-y_{n_{k}}^{i}, A_{i} y_{n_{k}}^{i}-A_{i} s_{n_{k}}\right\rangle \\
& +\left\langle x-y_{n_{k}}^{i}, A_{i} s_{n_{k}}\right\rangle \\
= & \left\langle x-y_{n_{k}}^{i}, A_{i} y_{n_{k}}^{i}-A_{i} s_{n_{k}}\right\rangle+\left\langle x-y_{n_{k}}^{i}, A_{i} s_{n_{k}}\right\rangle \\
\geq & \left\langle x-y_{n_{k}}^{i}, A_{i} y_{n_{k}}^{i}-A_{i} s_{n_{k}}\right\rangle+\left\langle x-y_{n_{k}}^{i}, \frac{s_{n_{k}}-y_{n_{k}}^{i}}{\lambda_{n}^{i}}\right\rangle .
\end{aligned}
$$

Since $\left\|y_{n}^{i}-s_{n}\right\| \rightarrow 0$ and $A_{i}$ is L-Lipschitz continuous,

$$
\lim _{n \rightarrow \infty}\left\|A_{i} y_{n}^{i}-A_{i} s_{n}\right\|=0
$$

Passing the limit in 29, as $k \rightarrow \infty$ and using $(30), y_{n_{k}}^{i} \rightarrow p$, we obtain $\langle x-p, y\rangle \geq 0$ for all $(x, y) \in G\left(Q_{i}\right)$. This together with the maximal monotonicity of $Q_{i}$ implies that $p \in Q_{i}^{-1} 0=V I\left(A_{i}, F\right)$ for all $1 \leq i \leq N$. Hence, $p \in F$.

Finally, we show that $x_{n} \rightarrow p=x^{\dagger}:=P_{F} x_{1}$. From 18 and $x \in F$, we have

$$
\left\|x_{n}-x_{1}\right\| \geq\left\|x^{\dagger}-x_{1}\right\|, \quad \forall n \geq 0 .
$$

This relation together with the lower weak semi-continuity of the norm implies that

$$
\left\|x^{\dagger}-x_{1}\right\| \leq\left\|p-x_{1}\right\| \leq \liminf _{k \rightarrow \infty}\left\|s_{n_{k}}-x_{1}\right\| \leq \limsup _{k \rightarrow \infty}\left\|s_{n_{k}}-x_{1}\right\| \leq\left\|x^{\dagger}-x_{1}\right\| .
$$

By the definition of $x^{\dagger}, p=x^{\dagger}$ and $\lim _{n \rightarrow \infty}\left\|x_{n_{k}}-x_{1}\right\|=\left\|x^{\dagger}-x_{1}\right\|$. Thus, from $x_{n_{k}}-x_{1}-x^{\dagger}-x_{1}$ and Lemma (Kadec-Klee) we obtain $x_{n_{k}}-x_{1} \rightarrow x^{\dagger}-x_{1}$, and so $x_{n_{k}} \rightarrow x^{\dagger}$. Lemma 2.3 ensures that the sequences $\left\{y_{n}^{i}\right\},\left\{z_{n}^{i}\right\}$ also converge strongly to $P_{F} x_{1}$.

\section{Application to Signal Recovery}

Signal processing is analysis, modifying, and synthesizing signals. We can use signal processing techniques for improving transmission, storage efficiency and subjective quality and also emphasizing or defecting components of interest in a measured signal. Signal processing problem can be modeled as the following under determinate linear equation system $b=B x+\nu$ where $x$ is a original signal with $N$ components to be recovered $\left(x \in \mathbb{R}^{N}\right), \nu, b$ are noise and the observed signal with noisy for $M$ components respectively $\left(\nu, b \in \mathbb{R}^{M}\right)$ and $B: \mathbb{R}^{N} \rightarrow \mathbb{R}^{M}(M \leq N)$ is a filtering. Finding the solutions of $b=B x+\nu$ can be seen as solving least squares (LS) problem

$$
\min _{x \in \mathbb{R}^{N}} \frac{1}{2}\|b-B x\|_{2}^{2}
$$


where $\|$.$\| is l_{2}-$ norm defined by $\|x\|=\sqrt{\sum_{i=1}^{n}\left|x_{i}\right|^{2}}$. The solution of 31 can be estimated by many well known iteration methods [13, 45]. Many algorithms based on optimization have been proposed for solving signal recovery problems 31 , see in [22, 27, 28]

In the real, the observation of signal may be disturbed by some filters and noises. The goal in this paper is to find the original signal without knowing the type of filter and noise. Thus, we can consider this problem in the following problem system.

$$
\min _{x \in \mathbb{R}^{N}} \frac{1}{2}\left\|B_{1} x-b_{1}\right\|_{2}^{2}, \min _{x \in \mathbb{R}^{N}} \frac{1}{2}\left\|B_{2} x-b_{2}\right\|_{2}^{2}, \ldots, \min _{x \in \mathbb{R}^{N}} \frac{1}{2}\left\|B_{N} x-b_{N}\right\|_{2}^{2},
$$

where $x$ is an original signal, $B_{i}$ is a bounded linear operator and $b_{i}$ is an observed signal with noisy for all $i=1,2, \ldots, N$. We can apply the Algorithm 2.1 to solve the problem 32 by setting $A_{i} x=B_{i}^{T}\left(B_{i} x-b_{i}\right)$ for all $i=1,2, \ldots, N$ and $C=\mathbb{R}^{N}$.

Algorithm 3.1. Initialization: Take $\rho>0, \mu \in(0,1), \theta \in[0,1)$ and $\left\{\theta_{n}\right\} \subseteq[0, \theta]$. Select arbitrary points $x_{0}, x_{1} \in H$. For $i=1,2, \ldots, N$ set $n:=1$

Step 1. Compute $S_{n}$,

$$
s_{n}=x_{n}+\theta_{n}\left(x_{n}-x_{n-1}\right)
$$

Step 2. Compute $y_{n}$,

$$
y_{n}^{i}=P_{C}\left(s_{n}-\lambda_{n}^{i} B_{i}^{T}\left(B_{i} s_{n}-b_{i}\right)\right),
$$

where $\lambda_{n}^{i}=\rho^{l^{i}}$ and $l^{i}$ is the smallest nonegative integer such that

$$
\rho^{l^{i}} \| B_{i}^{T}\left(B_{i}\left(s_{n}-y_{n}^{i}\right)\|\leq \mu\| s_{n}-y_{n}^{i} \| .\right.
$$

Step 3. Compute $z_{n}^{i}$,

$$
z_{n}^{i}=P_{T_{n}^{i}}\left(s_{n}-\lambda_{n}^{i} B_{i}^{T}\left(B_{i} y_{n}^{i}-b_{i}\right)\right), i=1, \ldots, N,
$$

where $\left.T_{n}^{i}=\left\{v \in H:\left\langle s_{n}-\lambda_{n}^{i} B_{i}^{T}\left(B_{i} s_{n}-b_{i}\right)\right)-y_{n}^{i}, v-y_{n}^{i}\right\rangle \leq 0\right\}$.

Step 4. Compute $\bar{z}_{n}$, i.e.,

$$
\bar{z}_{n}=\operatorname{argmax}\left\{\left\|z_{n}^{i}-s_{n}\right\|: i=1, \ldots, N\right\} .
$$

Step 5. Compute $x_{n+1}=P_{C_{n} \cap Q_{n}} x_{1}$, where

$$
C_{n}=\left\{v \in H:\left\|\bar{z}_{n}-v\right\| \leq\left\|s_{n}-v\right\|\right\},
$$

and

$$
Q_{n}=\left\{v \in H:\left\langle v-x_{n}, x_{n}-x_{0}\right\rangle \geq 0\right\} .
$$

Step 6. Set $n:=n+1$ and back to Step 1 .

In this experiment, the parameters $\rho_{n}, \theta_{n}$, and $\mu$ on an implemented algorithm in solving the image deblurring is set as equation (7). The Cauchy error and the signal error are measured by using second norm $\left\|x_{n}-x_{n-1}\right\|_{2}$ and $\left\|x_{n}-x\right\|_{2}$ respectively. The performance of the proposed method at $n^{\text {th }}$ iteration is measured quantitatively by the means of the signal-to-ratio (SNR), which is defined by

$$
\operatorname{SNR}\left(x_{n}\right)=20 \log _{10}\left(\frac{\|x\|_{2}}{\left\|x_{n}-x\right\|_{2}}\right)
$$

where $x_{n}$ is the recovered signal at $n^{\text {th }}$ iteration by using the proposed method.

The original signal $x$ with $N=256, M=128$ is generated by the uniform distribution in the interval $[-2,2]$ with $m=40$ nonzero element. The matrix $B_{1}, B_{2}$ and $B_{3}$ are generated by the Gaussian matrix generated by the MATLAB routine $\operatorname{randn}(M, N)$. The observation $b_{1}, b_{2}$ and $b_{3}$ with $M=128$ are generated by white Gaussian noise with signal-to-noise ratio $S N R=20$ (For $\left.B_{1}\right), S N R=40\left(\right.$ For $\left.B_{2}\right)$ and $S N R=30\left(\right.$ For $\left.B_{3}\right)$, respectively. The process is started with signal initial data $x_{1}$ with $N=256$ are picked randomly. 

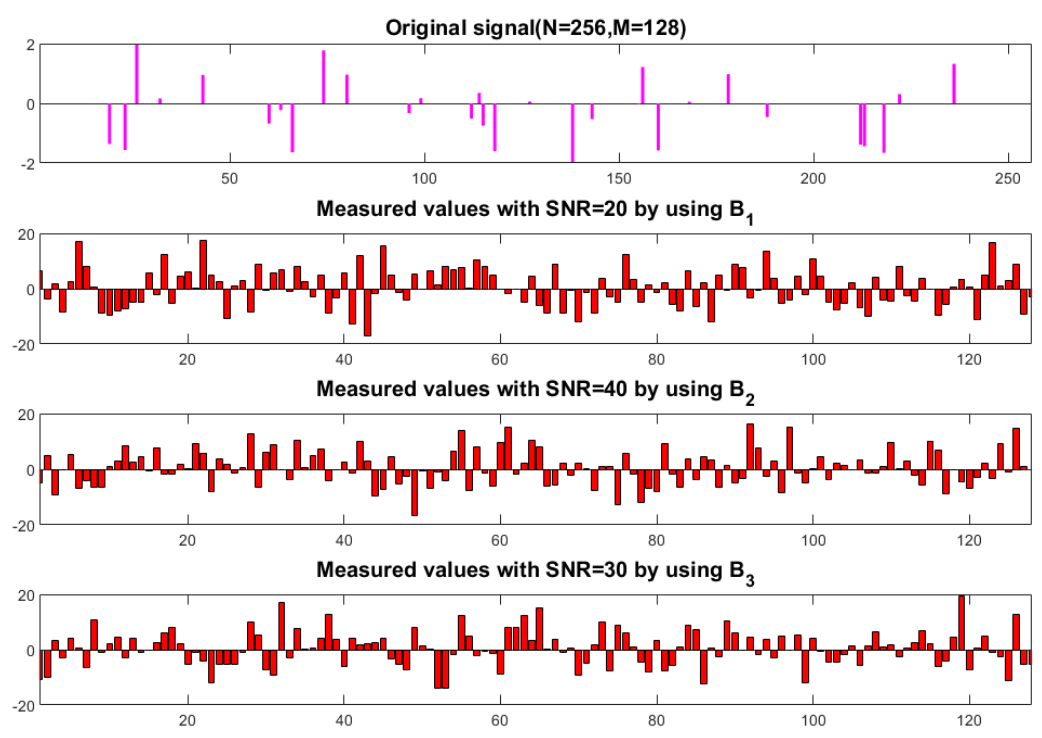

Figures 1-4: The original signal, observation data using $S N R=20\left(\right.$ For $\left.B_{1}\right), S N R=40\left(\right.$ For $\left.B_{2}\right)$ and $S N R=30\left(\right.$ For $\left.B_{3}\right)$, respectively.

Next, we aim to find the solutions of signal recovery problem $(32)$ with $\mathrm{N}=1$ by using the our Algorithm 3.1. We show the performance of $B_{1}, B_{2}$ and $B_{3}$ with $\mathrm{N}=256, \mathrm{M}=128$.
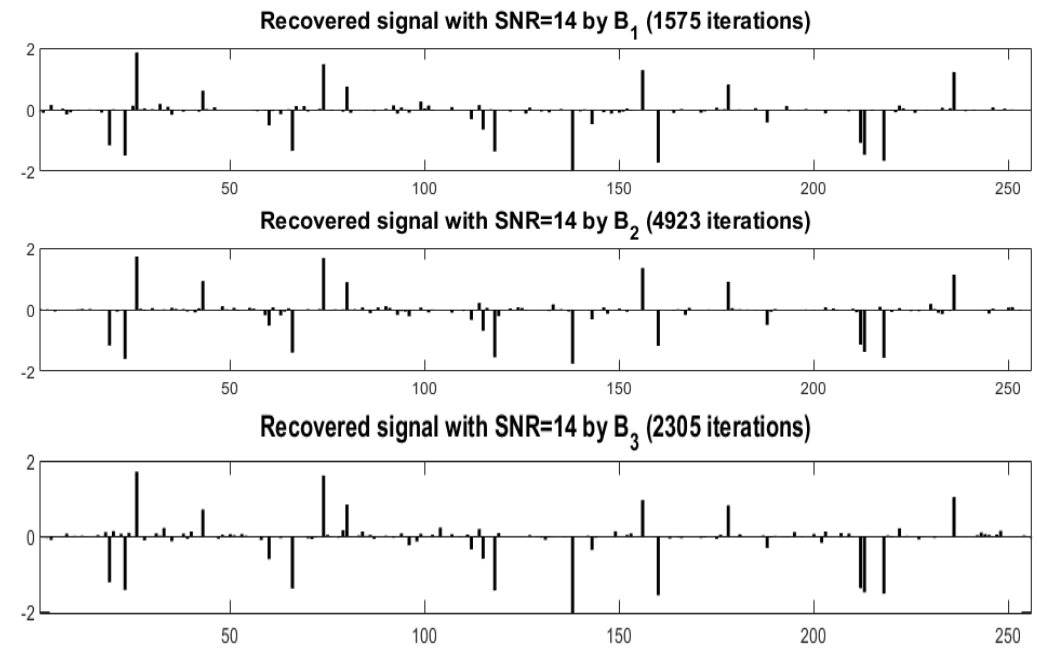

Figures 5-7 : Recovering Signal based on SNR $=14$ quality by $B_{1}, B_{2}$ and $B_{3}$.

Next, we aim to find the solutions of signal recovery problem (31) with $\mathrm{N}=2$ by using Algorithm 3.1. We show the performance of $B_{1}, B_{2}$ and $B_{1}, B_{3}$ and $B_{2}, B_{3}$ with $\mathrm{N}=256, \mathrm{M}=128$.

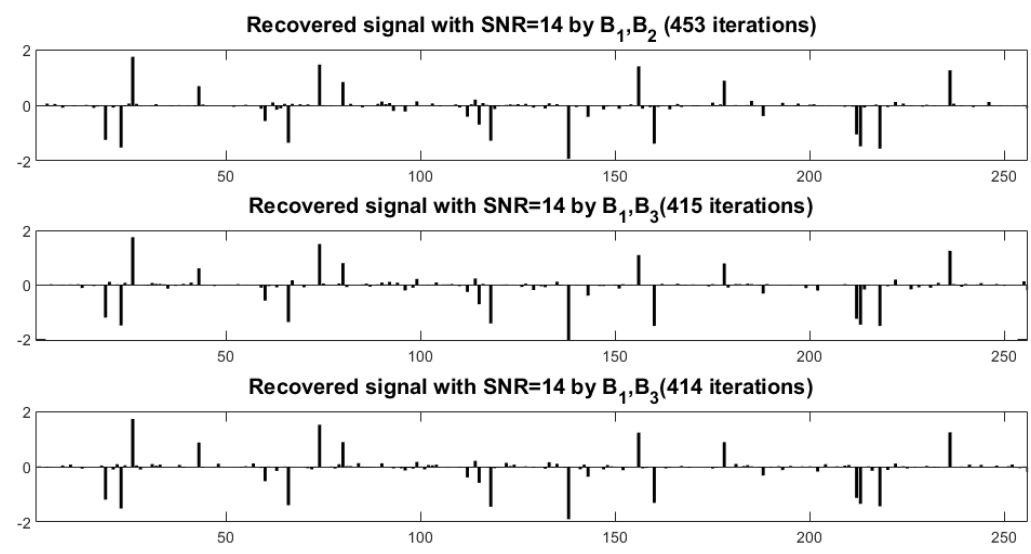


Figures 8-10 : Recovering Signal based on SNR $=14$ quality by $B_{1}, B_{2}$ and $B_{1}, B_{3}$ and $B_{2}, B_{3}$.

Next, we aim to find the solutions of signal recovery problem (31) with $\mathrm{N}=3$ by using Algorithm 3.1. We show the performance of $B_{1}, B_{2}, B_{3}$ with $\mathrm{N}=256, \mathrm{M}=128$.

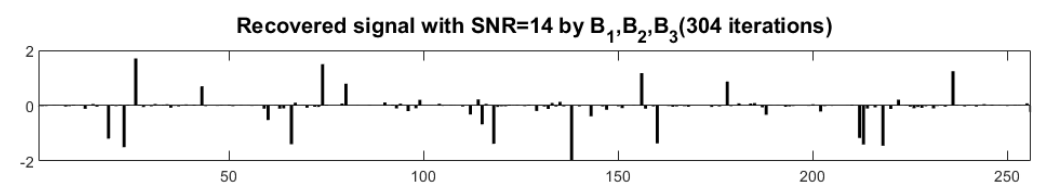

Figure 11 : Recovering Signal based on SNR $=14$ quality by $B_{1}, B_{2}, B_{3}$.

The Cauchy error, signal error and SNR quality of the proposed method for recovering the degraded signal are shown in Figures 12-14. The Cauchy error shows that the proposed method can be applied to signal recovering problem. And, the signal error confirms the convergence of the implemented algorithm.
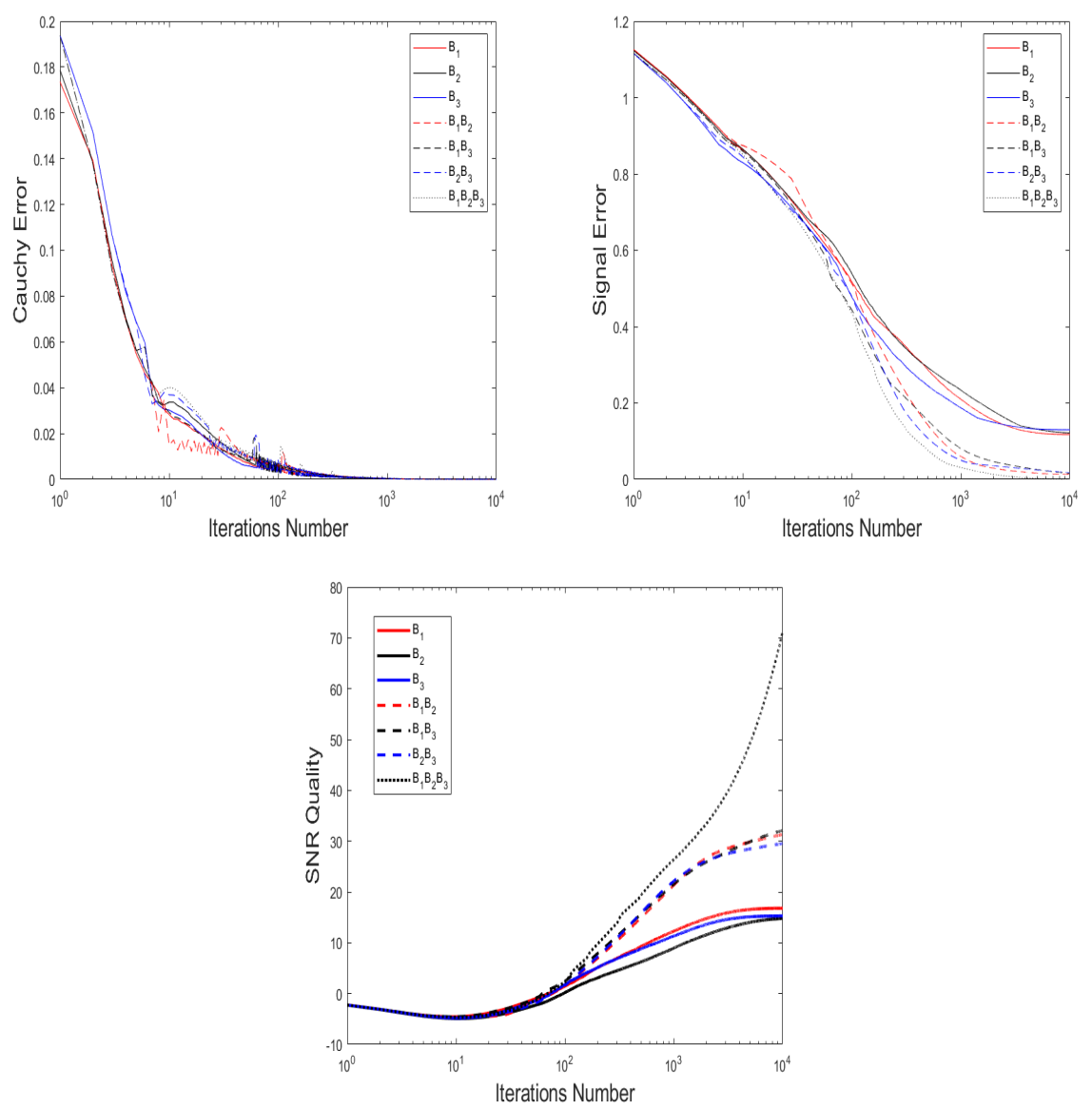

Figures 12-14 Cauchy Error, Signal Error and SNR Quality of the proposed methods in recovering the observed signal.

It is clearly seen that the solution of the signal recovering problem solved by the proposed algorithm get the quality improvements of the observed signal.

\section{Application to Image Recovery Problem}

Image restoration is the process of recovering an unknown image by denoising and deblurring of image. The image restoration problem can be considered in the following linear equation system: 


$$
b=B x+v,
$$

where $x \in \mathbb{R}^{n \times 1}$ is an original image, $b \in \mathbb{R}^{m \times 1}$ is the unknown image which is by blurred by matrix $B \in \mathbb{R}^{m \times n}$ and added by noise $v$. One technique in order to solve problem 33 is the inverse filtering when the image is blurred by a know blurring matrix $B$ some case the inverse of blurring matrix $B$ is difficult to fined, the convex, minimization is use, which is known as the following least squares (LS) problem (31).

In the real, we do not know the blurring matrix of any unknown image in general. So, the goal of solving image restoration is deblurring the image without knowing which is in the blurring operator. This problem can be considered in the problem system 32 where $x$ is the original true image, $B_{i}$ is the blurred matrix, $b_{i}$ is the blurred image by the blurred matrix $B_{i}$ for all $i=1,2, \ldots, N$. We know that $B_{i}^{T}\left(B_{i} x-b_{i}\right)$ is Lipschitz continuous for each $i=1,2, \ldots, N$, thus we can apply our Algorithm 3.1 to solve the problem 32 in the area of image restoration problem.

For showing the advantage of our Algorithm (3.1), we will use the following different three types of blurred matrices:

(1) Gaussian blur of filter size $9 \times 9$ with standard deviation $\mathrm{s}=4\left(B_{1}\right)$.

(2) Out of focus blur (Disk) with radius $\mathrm{r}=6\left(B_{2}\right)$.

(3) Motion blur specifying with motion length of 21 pixels $($ len $=21)$ and motion orientation $11^{\circ}(\theta=11)\left(B_{3}\right)$.

We will test these different three blur matrices with the following original Grey and RGB images.
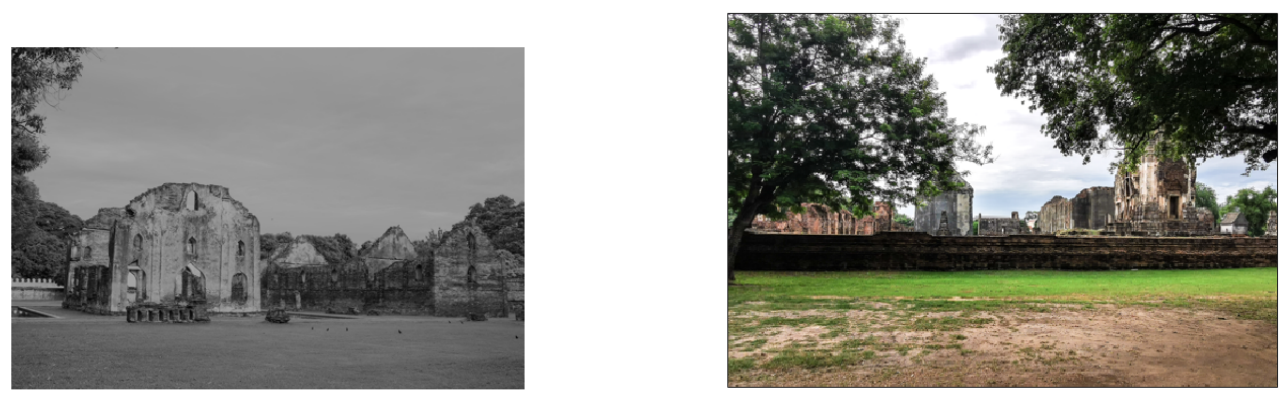

Figures 15-16 : The original Grey and RGB image of sizes $320 \times 480$ and $323 \times 475 \times 3$, respectively.

Three different types of blurred Grey and RGB images degraded by the blurring matrixes $B_{1}, B_{2}$ and $B_{3}$ are shown in Figures 17-22.

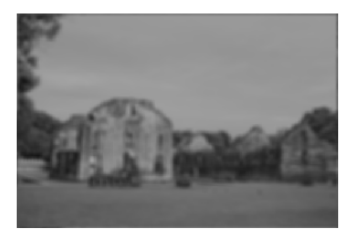

Gaussian Blurred Image

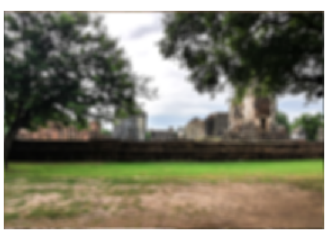

Gaussian Blurred Image

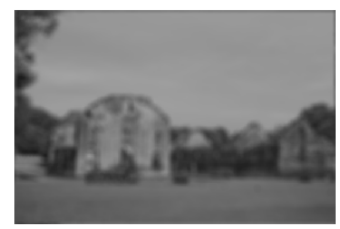

Out of Focused Image

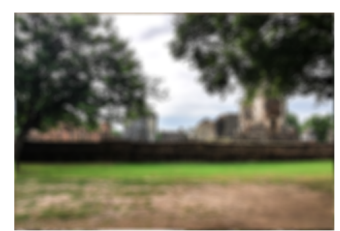

Out of Focused Image

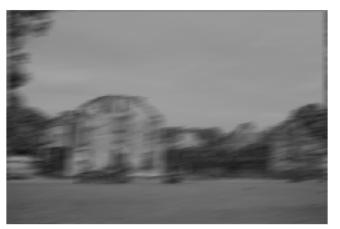

Motion Blurred Image

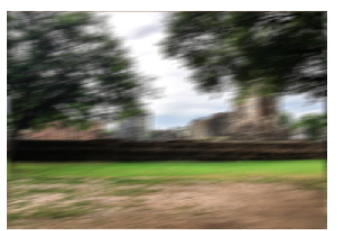

Motion Blurred Image

Figures 17-22 : Three degraded Grey and RGB images by blurred matrices $B_{1}, B_{2}$ and $B_{3}$, respectively. 
To show the first efficiency of our Algorithm 3.1 we put one by one of the blurring matrices $B_{1}, B_{2}$ and $B_{3}$ when $10000^{\text {th }}$ iterations is the stoping of the Algorithm:

Case I: Inputting $B_{1}$ on the Algorithm 3.1.

Case II: Inputting $B_{2}$ on the Algorithm 3.1,

Case III: Inputting $B_{3}$ on the Algorithm 3.1;

are shown in Figures 23-28 that becomposed of the restored image and its PSNR.
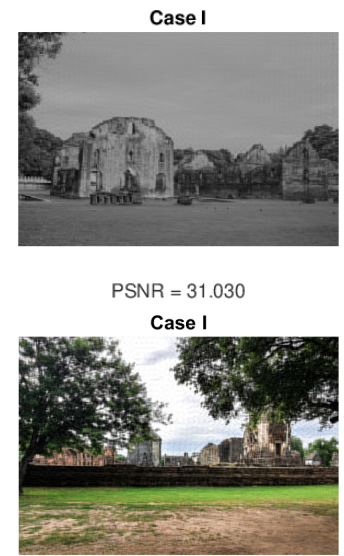

PSNR $=26.070$

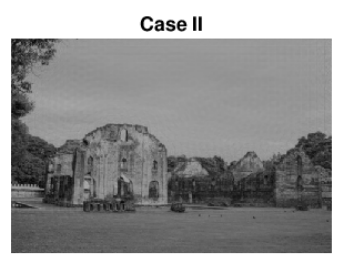

PSNR $=32.752$

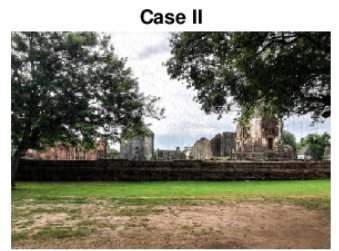

PSNR $=31.687$

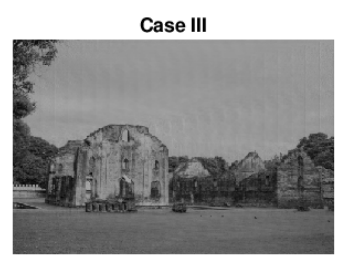

PSNR $=35.149$

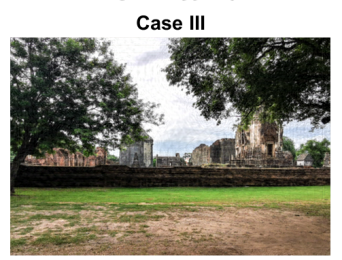

PSNR $=28.241$

Figures 23 -28 : The reconstructed Grey and RGB images with their PSNR for different three cases being used the proposed algorithm presented in $10000^{\text {th }}$ iterations, respectively.

Next, we put two different blurred matrixes into our Algorithm 3.1, so we can split testing into following there cases when $10000^{\text {th }}$ iterations is the stoping of the Algorithm:

Case IV: Inputting $B_{1}$ and $B_{2}$ on the Algorithm 3.1;

Case V: Inputting $B_{1}$ and $B_{3}$ on the Algorithm 3.1 .

Case VI: Inputting $B_{2}$ and $B_{3}$ on the Algorithm 3.1 .

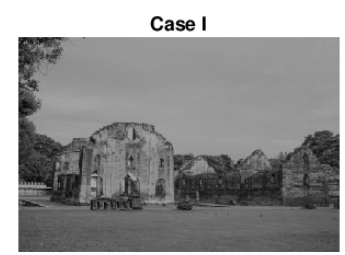

PSNR $=39.445$

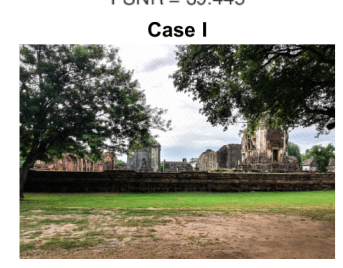

PSNR $=34.691$

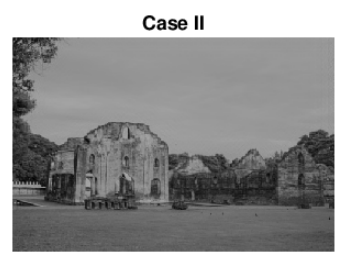

PSNR $=35.558$

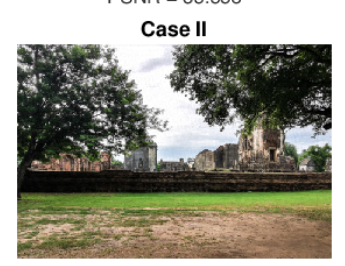

PSNR $=35.841$

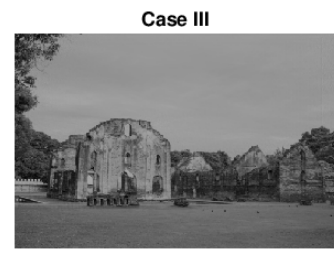

PSNR $=40.323$

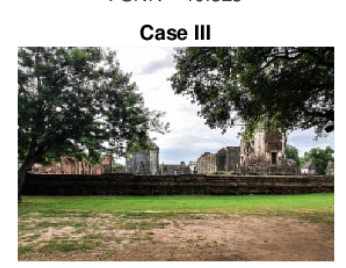

PSNR $=30.304$

Figures 29-34 : The reconstructed Grey and RGB images with their PSNR for different three cases being used the proposed algorithm presented in $10000^{\text {th }}$ iterations, respectively.

It can be seen from Figures 29-34 that the quality of restoration by using the Algorithm 3.1 when two different blurring matrixes are used $(\mathrm{N}=2)$ has improved compare with the previous result for every case, see on Figures 23-28.

The last case is inputting three different blurring matrixes $B_{1}, B_{2}$ and $B_{3}$ in Algorithm (3.1). The stopping of the algorithm is $10000^{\text {th }}$ iterations. The result are shown in the following figures. 


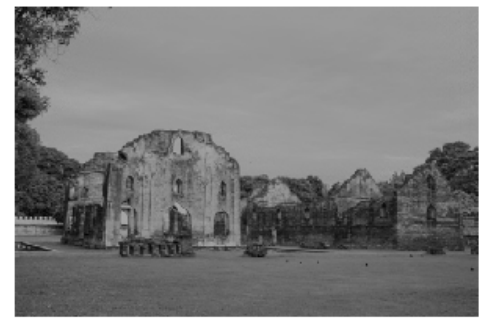

PSNR $=41.840$

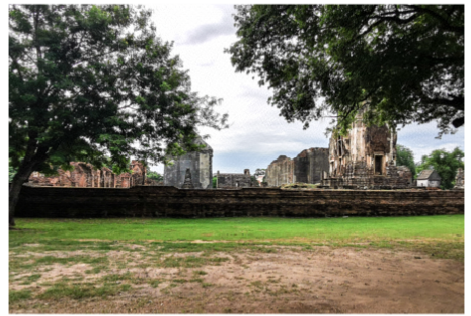

$\mathrm{PSNR}=36.732$

Figures 35-36 : The reconstructed Grey and RGB images from the blurring operators $B_{1}, B_{2}$ and $B_{3}$ being used the proposed algorithm presented in $10000^{\text {th }}$ iterations, respectively.

Figures 35-36 show the reconstructed Grey and RGB images with thousand iteration. It has been found that the quality of the recovered Grey and RGB images obtained by this algorithm is highest compared to the previous two algorithms.

The Cauchy error define as $\left\|x_{n}-x_{n-1}\right\|<10^{-5}$. The Figure error is defined as $\left\|x_{n}-x\right\|$ where $x$ is the original image. The performance of the proposed at $x_{n}$ on image restoring process is measured quantitatively by the means of the peak signal-to-noise ratio (PSNR), which is defined by

$$
\operatorname{PSNR}\left(x_{n}\right)=20 \log _{10}\left(\frac{255^{2}}{M S E}\right)
$$

where $M S E=\left\|x_{n}-x\right\|^{2},\left\|x_{n}-x\right\|$ is the second norm of $\operatorname{vec}\left(x_{n}-x\right)$.

The Cauchy error plot is shown for Algorithm 3.1 the validity while the Figures error plot is shown to confirms the convergence of the proposed method and the PSNR quality plot is shown for the measured quantitatively of the image.
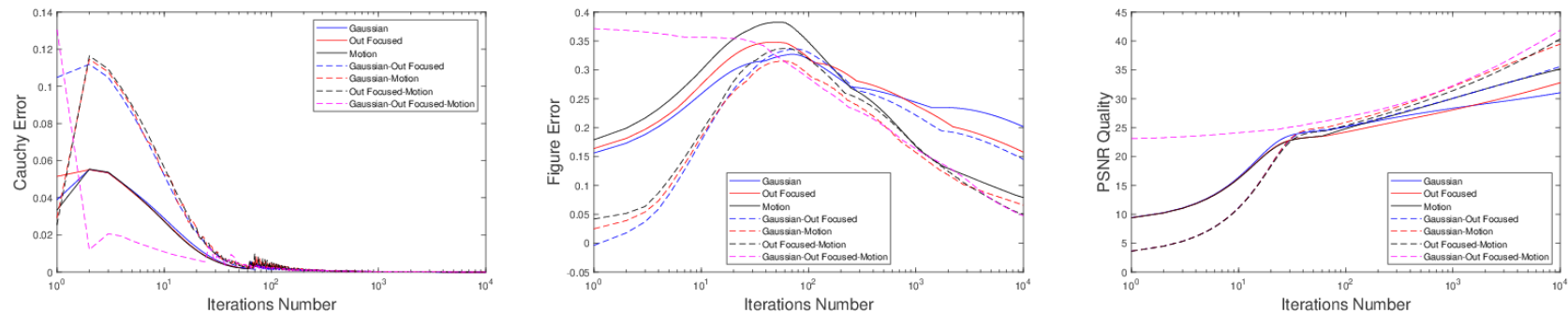

Figures 37-39 : Cauchy error, Figure error and PSNR quality plots of the proposed iteration in all cases of Grey images.
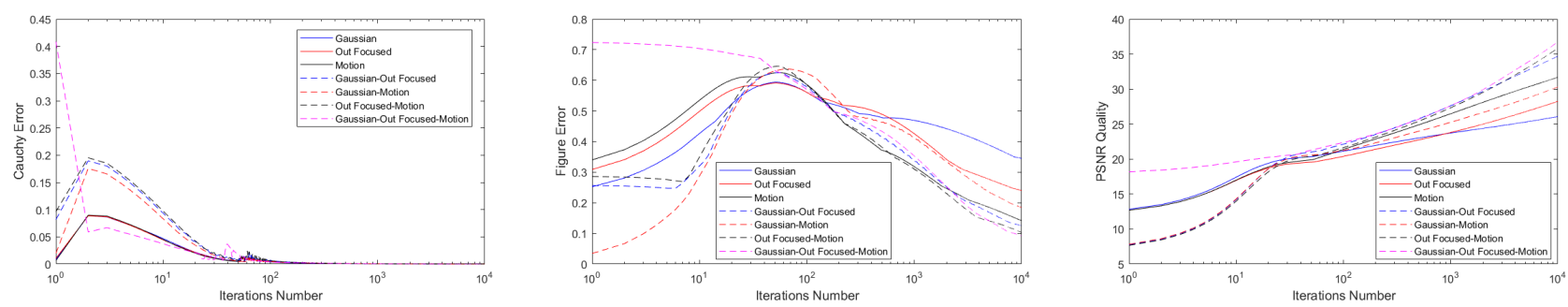

Figures 40-42 : Cauchy error, Figure error and PSNR quality plots of the proposed iteration in all cases of RGB images.

From Figures 37-42, it is clearly seen that the common solution of deblurring problem with $(N \geq 2)$ get the quality improvements of the reconstructed Grey and RGB images. Another advantage of the proposed method when the common solution of two or more image deblurring problem has been used to restored 
image is that the received image is more consistent than usual (See on Figures 43-56). Figures 43-56 show the reconstructed Grey and RGB images by using the proposed algorithm in getting the common solution of the following problem with the same PSNR.

(1) Deblurring by inputting $B_{1}, B_{2}$ and $B_{3}$ on the Algorithm 3.1, respectively.

(2) Deblurring by inputting $B_{1}$ and $B_{2}, B_{1}$ and $B_{3}, B_{2}$ and $B_{3}$ on the Algorithm 3.1 , respectively.

(3) Deblurring by inputting $B_{1}, B_{2}$ and $B_{3}$ on the Algorithm 3.1 .

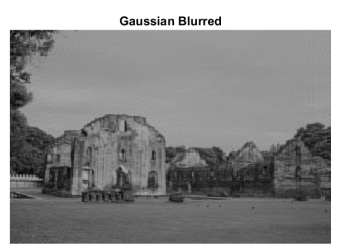

PSNR $=31\left(9779^{\text {hititeration })}\right.$

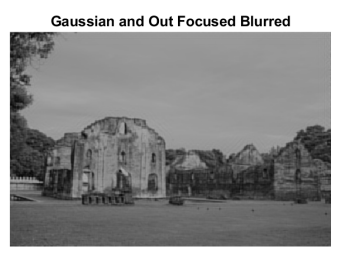

$\operatorname{PSNR}=31\left(1496^{\text {th }}\right.$ iteration $)$

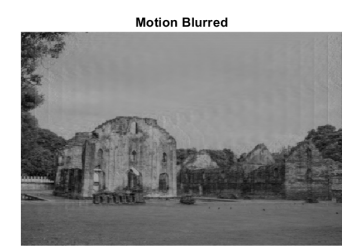

PSNR $=31\left(1493^{\text {th }}\right.$ iteration $)$

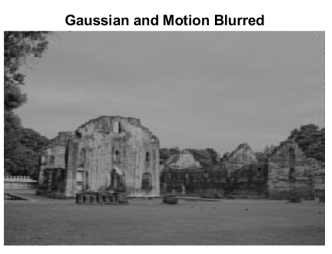

PSNR $=31\left(703^{\text {th }}\right.$ iteration $)$

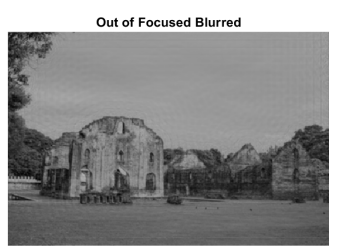

PSNR $=31\left(4535^{\left.t^{\text {th }} \text { iteration }\right)}\right.$

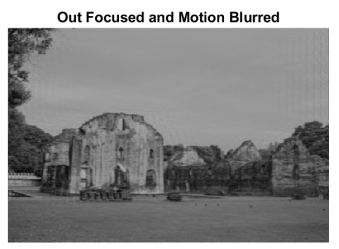

PSNR $=31\left(880^{\text {th }}\right.$ iteration $)$

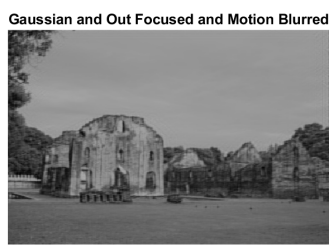

PSNR $=31\left(679^{\text {th }}\right.$ iteration $)$

Figures 43-49 : The reconstructed Grey images of all cases being used proposed Algorithm 3.1 with $\mathrm{PSNR}=31$.

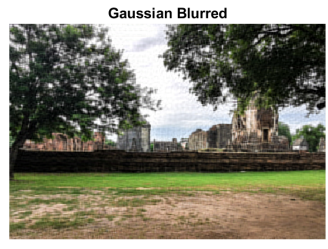

PSNR $=26\left(9420^{\text {th }}\right.$ iterlation $)$

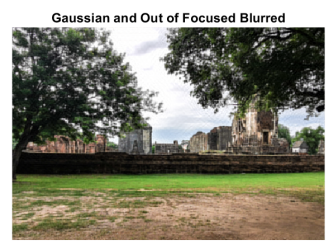

PSNR $=29\left(5883^{\text {th }}\right.$ teration $)$

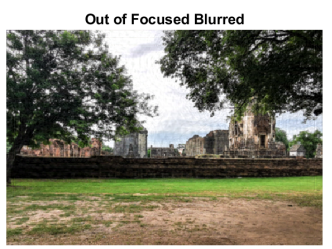

PSNR $=29\left(8937^{\text {th }}\right.$ Iteration $)$

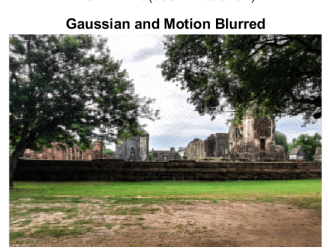

PSNR $=29\left(1596^{\text {th }}\right.$ Iteration $)$

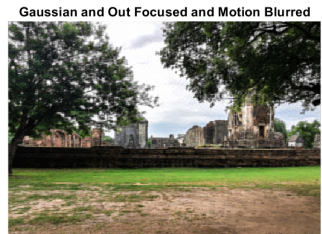

PSNR $=29\left(1542^{\left.\text {th }^{\text {iteration}}\right)}\right.$

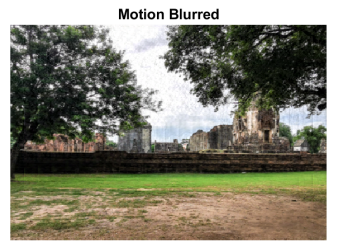

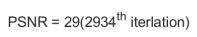

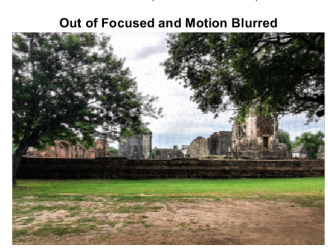

PSNR $=29\left(1713^{\text {th }}\right.$ iteration $)$

Figures 50-56 : The reconstructed RGB images of all cases being used proposed Algorithm 3.1 with $\mathrm{PSNR}=29$. 


\section{Conclusions}

In this paper, we solve common variational inequality problems by building the algorithm using the inertial technique with a parallel CQ subgradient extragradient method. We show the strong convergence of the algorithm under some suitable assumptions on the monotone and $L$ - Lipschitz continuous operator with constant $L$ is unknown. We also apply our proposed algorithm to solve signal and image recovery. We obtain that our algorithm gets increased efficiency when the subproblems are increasing in both signal and image recovery, see in Figures 5-14 (signal recovery) and Figures 23 - 56 (image recovery).

\section{Acknowledgement}

This research project was supported by the Thailand Science Research and Innovation Fund and the University of Phayao (Grant No. FF64-UoE002).

\section{References}

[1] Y. Alber, I. Ryazantseva, Nonlinear ill-posed problems of monotone type. Springer. London,(2006).

[2] N. Assad, W. Kirk, Fixed point theorems for set-valued mappings of contractive type. Pacific Journal of Mathematics. (1972), 43(3), 553-562.

[3] P. K. Anh, D. Van Hieu, Parallel hybrid iterative methods for variational inequalities, equilibrium problems, and common fixed point problems. Vietnam Journal of Mathematics. (2016), 44(2), 351-374,.

[4] P. K. Anh, D. Van Hieu, Parallel and sequential hybrid methods for a finite family of asymptotically quasi $\phi$-nonexpansive mappings. Journal of Applied Mathematics and Computing. (2015), 48(1-2), 241-263.

[5] H.H. Bauschke,J.M. Borwein, On projection algorithms for solving convex feasibility problems. SIAM review. (1996), 38(3), $367-426$.

[6] Y. Censor, W. Chen, P.L. Combettes, R. Davidi, G.T. Herman, On the effectiveness of projection methods for convex feasibility problems with linear inequality constraints. Computational Optimization and Applications, (2012), 51(3), 10651088.

[7] Y. Censor, A. Gibali, S. Reich, Strong convergence of subgradient extragradient methods for the variational inequality problem in Hilbert space. Optimization Methods and Software, (2011), 26(4-5), 827-845.

[8] Y. Censor, A. Gibali, S. Reich, The subgradient extragradient method for solving variational inequalities in Hilbert space. Journal of Optimization Theory and Applications. (2011), 148(2), 318-335.

[9] Y. Censor, A. Gibali, S. Reich, S. Sabach, Common solutions to variational inequalities. Set-Valued and Variational Analysis, (2012), 20(2), 229-247.

[10] W. Chuayjan, S. Thianwan, Strong convergence of a hybrid projection algorithm for equilibrium problems, variational inequality problems and fixed point problems in a Banach space. In Abstract and Applied Analysis (Vol. 2009). Hindawi.

[11] J. Deepho, W. Kumam, P. Kumam, A new hybrid projection algorithm for solving the split generalized equilibrium problems and the system of variational inequality problems. Journal of Mathematical Modelling and Algorithms in Operations Research, (2014), 13(4), 405-423.

[12] C. Fang, S. Chen, Some extragradient algorithms for variational inequalities. Advances in variational and hemivariational inequalities, Adv. Mech Math., vol. 33. Springer, Cham (2015), 145-171.

[13] H. W. Engl, M. Hanke, A. Neubauer, Regularization of Inverse Problems., Dordrecht: Kluwer Academic Publishers, 2000.

[14] Eslamian, M.: Convergence theorems for nonspreading mappings and nonexpansive multivalued mappings and equilibrium problems. Optimization Letters, (2013), 7(3), 547-557.

[15] A. Gibali, A new non-Lipschitzian projection method for solving variational inequalities in Euclidean spaces. Journal of Nonlinear Analysis and Optimization: Theory and Applications, (2015), 6(1), 41-5.

[16] R. Glowinski, R. Tremolieres, J.L. Lions, Numerical analysis of variational inequalities. Elsevier, (2011).

[17] P. Hartman, G. Stampacchia, On some non-linear elliptic differential-functional equations. Acta mathematica, (1966), 115(1), 271-310.

[18] Jiang, L., Su, Y.: Weak convergence theorems for equilibrium problems and nonexpansive mappings and nonspreading mappings in Hilbert spaces. Commun. Korean Math. Soc, (2012), 27(3), 505-512.

[19] T. Jitpeera, I. Inchan, P. Kumam, A general iterative algorithm combining viscosity method with parallel method for mixed equilibrium problems for a family of strict pseudo-contractions, Journal of Applied Mathematics and Informatics, (2011), 29(34), 621-639.

[20] J.S. Jung, Convergence of approximating fixed pints for multivalued nonself-mappings in Banach spaces. Korean J. Math, (2008), 16(2), 215-231.

[21] T.H. Kim, H.K. Xu, Strong convergence of modified Mann iterations. Nonlinear Analysis: Theory, Methods and Applications, (2005), 61(1-2), 51-60. 
[22] D. Kitkuan, K. Muangchoo, A. Padcharoen, N. Pakkaranang, P. Kumam, A viscosity forward-backward splitting approximation method in Banach spaces and its application to convex optimization and image restoration problems. Computational and Mathematical Methods, (2020), 2(4), e1098.

[23] S. Komal, P. Kumam, A Modified Subgradient Extragradient Algorithm with Inertial Effects. Communications in Mathematics and Applications, (2019), 10(2), 267-280.

[24] G.M. Korpelevich, The extragradient method for finding saddle points and other problems. Ekonomikai Matematicheskie Metody, (1976).

[25] H.B. Liu, Convergence theorems for a finite family of nonspreading and nonexpansive multivalued mappings and equilibrium problems with application. Math. Appl, 3, (2013), 49-61.

[26] C. Martinez-Yanes, H.K. Xu, Strong convergence of the CQ method for fixed point iteration processes. Nonlinear Analysis: Theory, Methods and Applications, (2006), 64(11), 2400-2411.

[27] N. Pakkaranang, P. Kumam, V. Berinde, Y.I. Suleiman, Superiorization methodology and perturbation resilience of inertial proximal gradient algorithm with application to signal recovery. The Journal of Supercomputing, (2020), 76(12), 9456-9477.

[28] N. Pakkaranang, P. Kumam, Y.I. Suleiman, B. Ali, Bounded perturbation resilience of viscosity proximal algorithm for solving split variational inclusion problems with applications to compressed sensing and image recovery. Mathematical Methods in the Applied Sciences, (2020).

[29] P. Pietramala, Convergence of approximating fixed points sets for multivalued nonexpansive mappings. Commentationes Mathematicae Universitatis Carolinae, (1991), 32(4), 697-701.

[30] R.T. Rockafellar, On the maximality of sums of nonlinear monotone operators. Transactions of the American Mathematical Society, (1970), 149(1), 75-88.

[31] N. Shahzad, H. Zegeye, Strong convergence results for nonself multimaps in Banach spaces. Proceedings of the American Mathematical Society, (2008), 136(2), 539-548.

[32] N. Shahzad, H. Zegeye, On Mann and Ishikawa iteration schemes for multi-valued maps in Banach spaces. Nonlinear Analysis: Theory, Methods and Applications, (2009), 71(3-4), 838-844.

[33] Y. Song, H. Wang, Convergence of iterative algorithms for multivalued mappings in Banach spaces, Nonlinear Anal. (2009), $70,1547-1556$.

[34] Y. Shehu, O.S. Iyiola, Strong convergence result for monotone variational inequalities. Numerical Algorithms, (2016).

[35] M.V. Solodov, B.F. Svaiter, A new projection method for variational inequality problems. SIAM J. Control Optim. (1999), 37, $765-776$.

[36] M.V. Solodov, B.F. Svaiter, Forcing strong convergence of proximal point iterations in Hilbert space. Math. Progr. (2000), 87, 189-202.

[37] H. Stark, Image Recovery Theory and Applications. Academic, Orlando, (1987).

[38] W. Takahashi, Nonlinear Functional Analysis. Yokohama Publishers, Yokohama, (2000).

[39] T. Thianwan, Strong Convergence of a General Iterative Algorithm for Mixed Equilibrium, Variational Inequality and Common Fixed Points Problems. (2013), 3(1), Article ID:27360.

[40] T. Thianwan, A general iterative algorithm for equilibrium problems and variational inequality problems in a Hilbert space. Asian-European Journal of Mathematics, (2010), 3(04), 685-705.

[41] R. Tibshirani, Regression shrinkage and selection via the Lasso, J.R. Stat. Soc. Ser B, (1996), 58, 267-288.

[42] D. Van Hieu, Parallel and cyclic hybrid subgradient extragradient methods for variational inequalities. Afrika Matematika, (2017), 28(5-6), 677-692.

[43] D. Van Hieu, P.K. Anh, Modified hybrid projection methods for finding common solutions to variational inequality problems. Computational Optimization and Applications, (2017), 66(1), 75-96.

[44] D. Van Hieu, Parallel hybrid methods for generalized equilibrium problems and asymptotically strictly pseudocontractive mappings. Journal of Applied Mathematics and Computing, (2017), 53(1-2), 531-554.

[45] C.R. Vogel, Computational Methods for Inverse Problems. PA: SIAM Philadelphia, (2002).

[46] N. Wairojjana, N. Pakkaranang, N. Pholasa, Strong convergence inertial projection algorithm with self-adaptive step size rule for pseudomonotone variational inequalities in Hilbert spaces. Demonstratio Mathematica, 54(1), (2021), 110-128.

[47] N. Wairojjana, M. Younis, N. Pakkaranang, N. Pholasa, Modified Viscosity Subgradient Extragradient-Like Algorithms for Solving Monotone Variational Inequalities Problems. Axioms, (2020), 9(4), 118.

[48] Z.M. Wang, S.Y. Cho, Y. Su, Convergence theorems basedonthe shrinking projection method for hemi-relatively nonexpansive mappings, variational inequalities and equilibrium problems. Thai Journal of Mathematics, (2017), 15, 835-860.

[49] Y. Yao, Y.C. Liou, Weak and strong convergence of Krasnoselski-Mann iteration for hierarchical fixed point problems. Inverse Probl. 24, Article ID 015015. (2008).

[50] I. Yamada, The hybrid steepest descent method for the variational inequality problem over the intersection of fixed point sets of nonexpensive mappings. In: Butnariu, D., Censor, Y., Reich, S. (eds.) Inherently Parallel Algorithms in Feasibility and Optimization and Their Applications, Elsevier, Amsterdam, (2001), pp. 473-504.

[51] S.E. Yimer, P. Kumam, A.G. Gebrie, R. Wangkeeree, Inertial Method for Bilevel Variational Inequality Problems with Fixed Point and Minimizer Point Constraints. Mathematics. (2019), 7, 841.

[52] J. Zhao, Q. Yang, Self-adaptive projection methods for the multiple-sets split feasibility problem. Inverse Problems, 035009 (2011), 27(3). 